\title{
The Biopsychosocial Model and the Sex-Positive Approach: an Integrative Perspective for Sexology and General Health Care
}

\author{
Filippo Maria Nimbi ${ }^{1}\left[\right.$ [ Roberta Galizia ${ }^{1} \cdot$ Roberta Rossi $^{2} \cdot$ Erika Limoncin $^{3} \cdot$ Giacomo Ciocca $^{1} \cdot$ Lilibeth Fontanesi $^{4}$. \\ Emmanuele Angelo Jannini ${ }^{3}$. Chiara Simonelli ${ }^{1} \cdot$ Renata Tambelli ${ }^{1}$
}

Accepted: 13 September 2021 / Published online: 22 September 2021

(c) The Author(s) 2021

\begin{abstract}
Introduction The Biopsychosocial model (BPS) represents a comprehensive paradigm of consolidated practices. Although it has been outlined as the gold standard in sexology, its implementation is often challenging. The sex-positive approach is a ground-breaking movement that is gaining popularity all over and is focused on the recognition of different sexual expressions as valid, consensual, healthy, and meaningful. However, a pragmatic implementation of this approach is still missing. Methods A critical review was conducted based on bibliographic research on Medline, PubMed, EBSCO, Cochrane Library, Scopus and Web of Science on relevant articles published from January 2011 to July 2021.

Results A total of 116 papers were included in the following review, indicating an increasing body of research about BPS and sex-positive frameworks during the last 10 years. One of the main limitations in the BPS is the scarce attention paid to socio-cultural factors involved in sexual expressions, such as the role of negative attitudes towards sexuality that may affect health care professionals' work. An application of the sex-positive approach to the BPS model may bring greater attention to the needs, values and desires of the individual, as well as allow a new knowledge and understanding of sexuality within a broader spectrum, including diversities and pleasures.

Conclusions The sex-positive approach represents a viable path that entails the willingness of health care professionals to get involved actively; criticize their personal attitudes, beliefs, and knowledge about sex and work hard to improve their practice in sexology.

Policy Implications A discussion of the possible fruitful integration between the BPS and the sex-positive approach is presented, highlighting practical applications in research, clinical practice, training and sex education and giving possible directions for future studies and policies.
\end{abstract}

Keywords Biopsychosocial model $\cdot$ Sex positive $\cdot$ Clinical psychology $\cdot$ Medicine $\cdot$ Counselling $\cdot$ Sexology $\cdot$ Sexual medicine

Filippo Maria Nimbi

filippo.nimbi@uniroma1.it

1 Dept. of Dynamic, Clinical and Health Psychology, Sapienza University of Rome, Via degli Apuli 1, 00185 Rome, Italy

2 Institute of Clinical Sexology, Rome, Italy

3 Dept. of Systems Medicine, University of Rome Tor Vergata, Rome, Italy

4 Dept. of Psychological, Health and Territorial Science, University G. d'Annunzio of Chieti, Chieti, Italy

\section{Introduction}

The recent scientific blooming of sexology has fostered a deeper understanding about sexualities, sexual behaviour and sexual health from a holistic perspective (Kleinplatz, 2013). We hereby refer to sexology as a discipline comprising activities and practices such as education; clinical psychology; sexual medicine; public health; basic and applied research; behavioural, social and anthropological sciences and ethics (Kirana et al., 2013; Reisman et al., 2015). More recently, new applicative fields have been connected to sexology such as advocacy, human rights, genetics, systems medicine, systems sexology, informatics, engineering, 
economics and politics (Jannini, 2017; Kaplan, 2014; Moscatelli et al., 2020).

This complex and multifaceted scenario is generally summarized by the biopsychosocial (BPS) model (Berry \& Berry, 2013; Engel, 1977; Montorsi \& Althof, 2004). Most health care professionals have an intuitive sense of the BPS model as a general approach that states that health and illness are caused by the interplay of biological (genetics, physiology and pathology), psychological (thoughts, emotions and behaviours) and social (relational, socio-economical, environmental and cultural) factors (Berry \& Berry, 2013). The BPS approach implies that every sexual expression consists of an interaction among biological, psychological and social factors and that it aims to provide comprehensive treatments to increase personal satisfaction and quality of life (QoL; Jannini et al., 2010). The assessment of a sexual problem under the BPS model should explore predisposing, precipitating and maintaining factors and involve medical, sexual and psychosocial history taking, physical exams, lab tests (if needed) and counselling with the patient and partner (if any) to determine the related distress (Hatsichristou et al., 2016). An effective BPS implementation requires collaboration between physicians, psychologists and other health care figures and stakeholders. With a patient-centred focus, clinicians should be able to understand the specific needs of their patients and offer a holistic treatment tailored to their characteristics and needs (Simonelli et al., 2010).

Alongside the BPS, a more recent perspective is represented by the sex-positive approach (Bhugra et al., 2010; Glickman, 2000; Gruskin et al., 2019; Ivanski \& Kohut, 2017; Kimmers et al., 2015; Williams et al., 2013), an ideology that promotes the importance of being open-minded with respect to sexual and gender expressions, being nonjudgemental and respectful of personal autonomy, giving centrality to the need for asking/obtaining consent for any sexual behaviour, discussing different sexual expressions and behaviours, emphasizing the validity of a person's sexuality and focusing on health care and education consequences. The sex-positive movement criticizes some current practices in sexology and general health, sexual communication in media and social situations and advocates for access to continuous, age-appropriate and comprehensive sexual health information, education and treatments (Ivanski \& Kohut, 2017).

Discussions involving sex-positive perspectives have become increasingly popular in recent years on the Internet and social media. Many advocates support the benefits of sex-positive principles for sexual and general health, while others remain cautious about a more open and positive communication about sexuality. Given the scientific and general attention on this issue, it is crucial to evaluate benefits or drawbacks of sex positivity for sexual health. What is missing and urgent is a practical discussion of the implementation of sex-positive ideals in daily health practices (Ivanski \& Kohut, 2017).

The BPS represents a complex and comprehensive model of consolidated and shared practices that is becoming more widespread and accepted by those in the scientific field, but it is not free from practical limits and biases. One of the main limitations of the BPS application is the scarce attention paid to socio-cultural factors involved in sexual expressions. The sex-positive approach is a ground-breaking movement that is mainly focused on the recognition of individual sexual expressions as valid, consensual, healthy and meaningful. However, a systematic application of this approach in health care systems is still scarce. In light of these considerations, the current critical review aims to discuss a possible fruitful integration between the BPS model and the sex-positive approach through the presentation of practical applications in research, clinical practice, training and sex education and the provision of possible directions for future studies and policies.

\section{Methodology}

The review was based on bibliographic research on Medline, PubMed, EBSCO, Cochrane Library, Scopus and Web of Science on relevant articles published in the last 10 years (from January 2011 to February 2021). The main keywords used for research, including asterisks, were "Biopsychosocial" AND "Sex*," "Integrat*" AND "Sex*," "SexPositive," and "Culture" AND "Sex*." Additional research included "positive attitudes" AND "Sex*" and "Pleasure." References and article's citations were additionally reviewed to find other sources. Results were reviewed, and the most relevant papers were selected following the aims of the present work. Papers were all published in English and fulltext accessible. Attention was paid to papers discussing cultural evolution of sexology, gold standards, treatment algorithms, critical issues, current achievements and future goals for clinical sexology and sexual medicine. Studies that were excluded concerned the application of the BPS model to other branches of medicine or psychology without making an explicit or implicit reference to sexuality, and/ or they were not considered useful by the authors in a critical discussion of a possible application to the sex-positive approach. Study data and authors' positions were analysed to determine the state of the BPS model, a possible sexpositive integration, and to clarify the pragmatic challenges to clinical practice and research. A total of 116 documents were included in the present review, indicating an increasing body of research that adopts the BPS and sex-positive frameworks during the last 10 years (see Fig. 1). Results will be presented and discussed starting from a rationale of some BPS model limits, highlighting the sex-positive approach's 
Fig. 1 PRISMA flow diagram

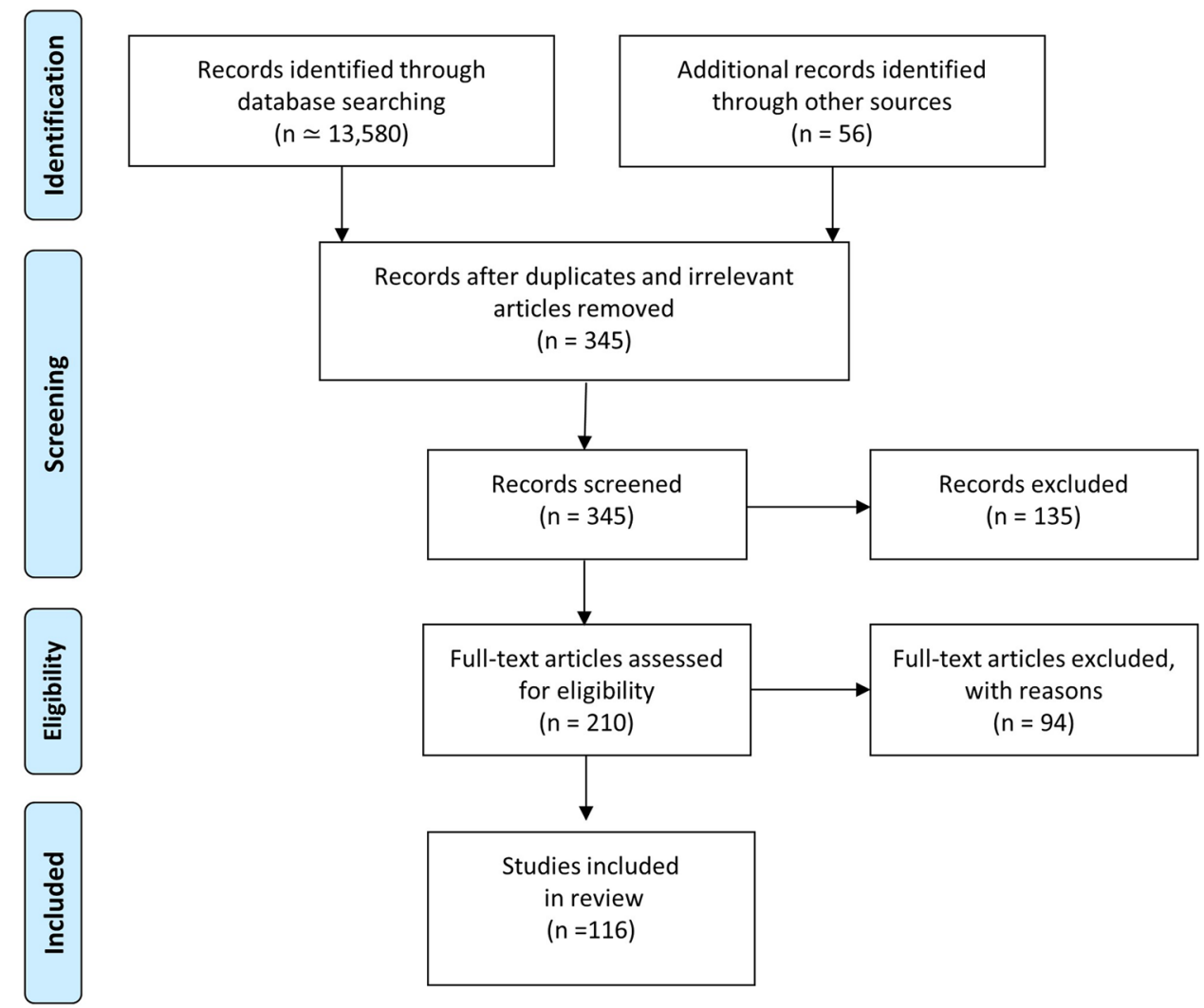

core values and discussing a possible application of the sexpositive approach to the BPS practice in research, training, clinical practice and sexual education.

\section{Results}

\section{Some Shadows of the BPS Model: When Culture and Attitudes are Neglected}

Although the BPS has been outlined as the gold standard in sexual medicine and clinical sexology (Berry \& Berry, 2013), it has been noted that the clinical implementation of BPS practice may be quite challenging (Simonelli et al., 2010). Some limits and implementation obstacles of the BPS approach have been highlighted in Table 1. The BPS requires multidisciplinary cooperation, eclecticism and flexibility, elements that still cannot be taken for granted in any social or health care context. Most of the time, medical and non-medical health care providers work separately, making it difficult to establish cross-collaboration for both clinical and research purposes (Perelman, 2015). It is essential for different specialists to work together to share aims and treatment algorithms and recognize the specificity of each professional figure (Kirana et al., 2013; Reisman et al., 2015). Knowledge and competence, as outcomes of good training and professional expertise, should lead to a greater flexibility in tailoring treatments to patients. Moreover, working in teams requires the ability to communicate between different professionals (e.g. physicians, psychologists, nurses, physiotherapists, GPs, etc.) who speak different technical languages (Althof, 2007; Jannini \& Reisman, 2019). In addition, several economic, financial and political issues must also be considered, such as service accessibility (mainly supplied in private practice), recognition of sexual health care providers and national and international accreditation of training standards. A BPS approach might be more expensive, and apparently longer if compared with unimodal treatments, but it usually reports better outcomes in clinical practice (Goldstein, 2012; Simonelli et al., 2010).

From a scientific perspective, the lack of data on validated protocols implementing BPS does not allow for the diffusion of consolidated procedures for sexual dysfunction management. Goldstein (2012) argued that health care professionals usually agree about the multidisciplinary nature of sexuality and the need for a BPS approach in patient care, but they find it difficult to put it into practice in the treatments. As a result, BPS practice is more often used during assessment and diagnosis instead of during treatment, a step in which unimodal procedures are still preferred.

One of the practical risks of the BPS implementation in sexology is that physicians frequently tend to underestimate 
Table 1 Limits of the biopsychosocial model (BPS) implementation in sexology

\begin{tabular}{|c|c|}
\hline se & $\begin{array}{l}\text { - Limited (but increasing) number of studies on sexuality under a BPS framework, especially evaluating integrative and } \\
\text { interdisciplinary treatments for sexual problems } \\
\text { - Relative scarcity of rigorous methods and reproducible studies } \\
\text { - Lack of randomized controlled trials (RCTs) and multicentred cross-country studies } \\
\text { - Available studies characterized by small sample size, lack of control groups, and long-term outcome measures } \\
\text { - Marginalized research on psycho-social factors of sexuality compared to bio-medical ones }\end{array}$ \\
\hline lini & $\begin{array}{l}\text { - Lack of evidence-based models of combined/integrated therapy for sexual dysfunctions } \\
\text { - Scarcity of data on efficacy of integrated treatments for sexual problems } \\
\text { - No information on long-term effects of integrated treatments for sexual problems } \\
\text { - Role of therapeutic skills and non-specific factors in treatments: "dodo-bird effect" (therapies have a positive effect on } \\
\text { patient, but is difficult to determine whether depending on methods, on individual clinician skills or on the fact that somec } \\
\text { is simply taking care of the problem) } \\
\text { - Flexibility between standard treatment protocols and patient-tailored therapy } \\
\text { - Predominance of medical/pathology-centred models on sexology clinical practice } \\
\text { - Patients' preference for medical and short treatments rather than psychological interventions } \\
\text { - Need of updated guidelines for clinicians to implement the BPS approach in their daily practice } \\
\text { - Low use of validated measures in clinical practice (e.g., questionnaires, interviews, etc.) }\end{array}$ \\
\hline rain & $\begin{array}{l}\text { - Lack of standardized and shared criteria for training courses both at the national and international level } \\
\text { - Lack of focus on socio-cultural aspects related to sexuality } \\
\text { - Lack of work on personal attitudes towards sex } \\
\text { - Supervisions and internships left to the personal sensitivity of the trainee (often not mandatory for the professional } \\
\text { diploma) } \\
\text { - Mono-professional boards in training courses (e.g., physicians train physicians, psychologists train psychologists) }\end{array}$ \\
\hline tical & $\begin{array}{l}\text { - Absence of international recognized criteria defining professional figures such as sex educators, sexual counsellors, sexual } \\
\text { therapists, etc } \\
\text { - No official recognition of "sexologist" as a real professional figure in many countries } \\
\text { - Marginalization of sexual health in financial and political acts }\end{array}$ \\
\hline
\end{tabular}

the assessment of psychological and relational factors (medical reductionism), while psychologists often fail to explore the biological side (psychological reductionism). Moreover, both professionals tend to generally undervalue the influence of societies and cultures in sexuality (Kirana et al., 2013). Therefore, the "S" of the BPS approach seems to have a limited space in the minds of clinicians despite many authors' arguments that beliefs, schemas and scripts are important factors in the onset of sexual dysfunctions and can play a significant role in understanding sexual behaviours, promoting adherence to treatments and improving clinician-patient relationships (Coyne et al., 2019; Maxwell et al., 2017; Nimbi et al., 2018, 2019; Tavares et al., 2020).

Sexuality occurs in a socio-cultural context, but clinicians are usually born and raised in the same cultural background of their patients (Atallah et al., 2016; Coleman et al., 2018). In this sense, some of the socio-cultural beliefs and expectations related to sexuality are shared among clinicians and patients and may be undervalued or neglected in the care. Due to the growth of migratory fluxes, meeting people of different cultures has become increasingly common in daily practice. Clinicians have numerous occasions to discuss sexual topics with patients from different geographical areas with specific cultures, ethnicities, religions, ethics and norms. Atallah et al. (2016) presented some important guidelines for creating sexological treatments that are respectful of cultural differences: clinicians are required to increase cultural sensitivity in their practice by examining the real weight of socio-cultural factors and dealing with them in their clinical work. As a result, there is the urgency of multi-centred and multicultural studies that could explain the role of cultural messages, stereotypes and beliefs in the way sexuality is expressed (Aumer, 2014; Neculăesei, 2015).

The socio-cultural issue in sexology is not limited to geographical differences: it refers, above all, to gender and sexual stereotypes and beliefs. Traditional sexual scripts are generally characterized by gender power inequality, penetration imperative and double standards. Adherence to these scripts is still common today, affecting sexual experiences and health (Klein et al., 2019). In this sense, it would be crucial to analyse how the clinical practice may be influenced by this range of beliefs and stereotype, for example, by determining health care providers' attitudes towards sex. Health care providers may report negative sexual attitudes based on gender, sexual identity, race/ethnicity, age and health conditions (Sabin et al., 2015). The presence of negative attitudes towards sex may hinder the clinicians' ability to promote sexual health (Klaeson et al., 2017). For example, 
many clinicians avoid asking their patients about sexuality although they recognize it to be among their professional goals (Frederick et al., 2018; Kotronoulas et al., 2009). The reasons why they often overlook patients' sexuality are varied, but include personal embarrassment, the belief that sexuality is not as important as the patient's main problem, insufficient sexological training or a concern about increasing patient's anxiety when asking about sex (Katz, 2005; Klaeson et al., 2017). As a result, patients rarely receive real holistic care that may include sexual health.

Many studies have explored the sexual attitudes of health professionals; available data in literature refer to specific health care figures and sexual topics. Clinician's negative attitudes towards gender identity and sexual orientation may predict their behavioural intention to discriminate others (Earnshaw et al., 2014; Vijay et al., 2018). Patients belonging to sexual minorities may avoid or delay medical treatment due to perceived discriminatory judgments by their health care professionals (Casagrande et al., 2007; Fisher et al., 2017). Tracy et al. (2010) highlighted that around $25 \%$ of lesbian patients delay their visits due to the fear of possible discrimination. As a result, lesbian women tend to ask for medical treatments less compared to heterosexual women and are less likely to access preventive care (Sabin et al., 2015)

Living in a sex-negative culture characterized by prejudices, stereotypes, recurrent use of labels and diverse forms of discrimination can also affect health in more subtle ways. On one hand, patients may hesitate to reveal their sexual orientation and gender identity and avoid reporting useful information about their sexual behaviour and health (e.g. being seropositive, having anal intercourse) to their health care professionals (Durso \& Meyer, 2013; Herek et al., 2009). On the other hand, physicians seem to be more used to recommend HIV testing and vaccination against hepatitis A and B to gay patients (based on sexual orientation) rather than assessing them for risky sexual behaviours (Petroll \& Mosack, 2011).

Clinicians' negative attitudes towards sex were also found regarding the elderly (Gewirtz-Meydan et al., 2018; Monteiro et al., 2017; Træen et al., 2019) and people with disabilities (Martino \& Perreault-Laird, 2019; Tamas et al., 2019), showing a widespread presence of conservative and denying positions on sexuality expressions of these varied groups. Negative attitudes can also be found towards pleasurable aspects in sexuality (Berdychevsky \& Carr, 2020). The "pleasure deficit" in sexual and reproductive health literature and practice may favour the idea that sex is a physiological act and should be regarded as emotionally neutral. Several treatments of male and female sexual dysfunction in sexual medicine have often put aside pleasure and satisfaction, favouring the primacy of function (Fahs, 2014; Grunt-Mejer, 2021; Levin, 2020). Moreover, clinicians may have prejudices against people with alternative erotic preferences and might feel uncomfortable and inadequate talking about clitoral stimulation, masturbation, female ejaculation, BDSM bondage-discipline/domination-submission/sadismmasochism), kinky practices and sex toys (Botta et al., 2019; Eichenberg et al., 2019; Hoff \& Sprott, 2009; Jannini et al., 2012; Kolmes et al., 2006; Miranda et al., 2019).

These are some of the most recent examples of studies showing that health care professionals are often emotionally and cognitively unprepared to deal with sexual health. It follows that clinicians (and society in a broader sense) should be sensitized and educated to develop a more realistic and positive conception of sexuality. To deliver scientific information in a non-judgmental way, practitioners need to take on the possible discomfort of discussing sexual health, sexual attitudes and more. In this regard, specific sexological training should overtake curriculum limitations, as well as personal and cultural prejudices and negative beliefs on sexuality (Parish \& Rubio-Aurioles, 2010; Tsimtsiou et al., 2006). Health care professionals generally receive few tools and knowledge regarding human sexuality within their standard training paths (Coleman et al., 2013), showing important gaps in specific areas of sexuality, such as sexual pain, arousal and desire (Althof \& Needle, 2011; Boyer et al., 2017). In this sense, implementing a sex-positive approach within the BPS model may be useful and urgently needed for giving deserved importance to the socio-cultural aspects of the BPS.

\section{Sex-Positive Approach}

First, Bullough (1976) described societies in terms of positive or negative sexual variance. Sex-negative societies usually encourage sexual abstinence (except for married couples with reproductive aims), and sex is largely constructed as being risky, problematic or taboo. Sex negativity is usually linked not only to prejudices associated with various sexual practices, but also to stigma towards women and other minorities (Glickman, 2000).

The concept of "sex-positive" has often been misinterpreted as simply being favourable to various kinds of sexual expression. However, a genuine sex-positive approach is more extensive; it emphasizes the pleasurable, rewarding and constitutional role of sexuality (Bhugra et al., 2010; Gruskin et al., 2019). The sex-positive approach finds its roots in authors such as Magnus Hirschfeld, Wilhelm Reich, Simone de Beauvoir, Shere Hite and Carol Queen and in years of social movements and feminist, queer, HIV/AIDS and anti-racism advocacy (Mosher, 2017). Starting from the experiences of many marginalized communities, the sex-positive approach aims to normalize a range of fluid sexual experiences. It recognizes the tremendous cultural diversity in sexual practices, acknowledging a substantial 
variation in personal meanings and preferences over time and space (Williams et al., 2013). The focus is primarily on the complexities of human interactions and thus not only on risks, deviance and pathology (Mosher, 2017). Being sexpositive means considering sexuality, including pleasure, as a natural, healthy and central part of human behaviour and well-being (Baggett et al., 2017; WAS, 2019). It focuses both on the absence of physical and emotional harm and risk reduction, as well as on the presence of pleasure and meaningful experiences. As highlighted by Kimmes et al. (2015), a positive sexual perspective considers all consensual sexual expressions to be valid and legitimate, stressing the importance of the subjective meaning that each person gives to their sexual well-being and health. In this sense, one person may opt for abstinence from sexual activity, whereas another may choose to engage in diverse and numerous sexual experiences with different partners. In both examples, a sex-positive approach recognizes the power of personal values and free self-determination without judging other experiences that are different from one's own.

The basic tools of the sex-positive approach are the promotion and dissemination of a comprehensive sex education and care aimed at every age group with diversified and personalized communication and content (Gruskin et al., 2019; Ivanski \& Kohut, 2017; Raymond \& Hutchison, 2019). For an easier understanding, the "sex-positive pillars" have been outlined in Table 2. A sex-positive approach critically questions the traditional understanding of erotic practices subject to heterosexism and monogamy-normative prejudices (Williams et al., 2013). Since human sexuality is a highly complex phenomenon that takes on different shades from person to person, it is necessary to talk about it separately from prejudices. Sex is not a different topic from others: when talking about sex in a low voice or looking around (signs of sexual negativity), one does nothing but reinforce marginalization and prejudices (Williams et al., 2013, 2015).

The sex-positive approach is somewhat similar to Positive Psychology (Williams et al., 2015): both are focused on building strengths and competences to improve the $\mathrm{QoL}$ and prevent discomfort and distress (Arakawa et al., 2013), moving from pathology-based models to ones of acceptance and empowerment (Burnes et al., 2017). Both recognize the importance of courage, curiosity, optimism, perseverance, compassion, pride and faith as protective factors from distress and pathology. People rely on their strengths to develop and express their sexual identity, to solve problems, to create intimacy (both with themselves and with their partners) and to be more satisfied and fulfilled (Williams et al., 2015).

In this sense, the sex-positive approach is totally consistent with the positions of the World Health Organization (WHO), the World Sexual Health Association (WAS) and the International Planned Parenthood Federation (IPPF) regarding sexual health and rights. For example, the WHO (2006) acknowledged that sexuality is shaped by the interactions of biological, psychological, cultural, social, economic, political, legal, ethical, historical and religious and spiritual factors. Each person is unique when considering the complex intersectionality among dimensions of human diversity (Williams et al., 2013). The WAS (2014) has provided the "Declaration of sexual rights," stating that "sexual health requires a positive and respectful approach to sexuality and sexual relationships, as well as the possibility of having pleasurable and safe sexual experiences, free of coercion, discrimination and violence." Sexual rights are respected when they are protected and promoted by principles such as equality and non-discrimination, prohibiting any distinction, exclusion or restriction (e.g. ethnic, racial, sexual and religious). This element has been also underlined in

Table 2 Sex-positive pillars

\begin{tabular}{ll}
\hline Pillar & Explanation \\
\hline Openness & $\begin{array}{l}\text { It empowers the curiosity towards sexuality, which allows an increase in personal and social knowledge, the possibility to discover } \\
\text { new satisfying ways to be sexual and the enhancement of discussions on sexuality and related topics in the community, with both } \\
\text { health care providers and within personal relationships }\end{array}$ \\
$\begin{array}{l}\text { Self-determination } \\
\text { Sexual self-determination is a combination of attitudes and abilities that lead people to set goals for themselves and to take } \\
\text { the initiative to reach these goals in their sexual experiences and health. It results in autonomy and freedom of choice } \\
\text { regarding reproduction, contraception, sexual expressions and behaviours }\end{array}$ \\
It guarantees equal access to the whole community to the best standards of comprehensive sex education, safer sex and harm \\
reduction practices in alignment with sexual health self-determination \\
Pleasure \\
It recognizes sexuality as a valid expression of pleasure, acknowledging that for many people, sexuality is not exclusively \\
a matter of reproduction and/or intimacy. Moreover, sexual motives can sensibly vary among individuals and within the \\
same person from day to day \\
It means actively agreeing to be sexual with someone at different moments and in varying forms. Consent is free from coercion \\
and revocable at any time. Sexual activity without consent is considered rape or sexual assault \\
Respect & It requires the recognition of differences in the expression of one's own and others' sexuality. It also involves acceptance and \\
equality of different forms of sexuality and promotes an active aversion to judgment and shaming (e.g. body shaming, "slut \\
shaming," homophobia, transphobia, HIV-stigma, sexism, etc.)
\end{tabular}


collaboration with the IPPF with a specific focus on young people (IPPF \& WAS, 2016). In presenting the key points of this approach, it is fundamental to consider the possible practical challenges of implementing a sex-positive approach within the BPS model in research, clinical work, professional training and education.

\section{Sex-Positive Application to BPS Practice}

A sex-positive perspective is already present in the scenario of good sexological procedures, but it has been taken for granted too often in daily practice. Professionals should therefore focus not only on sexual health prevention, such as reducing risky behaviours, but also stress the promotion of a positive view of sexuality (Williams et al., 2015). Sex as leisure is a novel (and potentially uncomfortable) idea to sexual health professionals as well as to the public. Some might dismiss it, but others would be open to the potential of this approach in addressing stereotypical misperceptions about sex. Failure to leverage this potential is reckless as it may lead to sub-optimal or ineffective sex-related policies, programmes and treatments (Berdychevsky \& Carr, 2020). According to this position, how can health care practitioners offer the best and most effective care in the field of sexual health? More specifically, how can BPS and sex-positive approaches be implemented in current sexual health systems? The answer is complex and regards different levels of integration.

\section{Research}

Most of the sex-positive literature published in the last few decades has been generated outside of the clinical (medical and psychological) fields, mainly in social work and sociology, women's and gender studies and law (Burnes et al., 2017; Glickman, 2000; Williams et al., 2013). Arakawa et al. (2013) highlighted a lack of research on the positive aspects of sexuality in sexology academic journals, showing that only 7\% of the articles published from 1960 to 2010 in four of the main sexology journals (The Journal of Sex Research, Archives of Sexual Behavior, The New England Journal of Medicine and Obstetrics and Gynaecology) focused on positive aspects of sexuality, while $35 \%$ and $58 \%$ of the papers embodied neutral and negative perspectives, respectively. Hargons et al. (2017) run a content analysis of sexuality research in counselling psychology journals (Journal of Counseling Psychology and The Counseling Psychologist), highlighting that out of 188 articles, only $4.78 \%$ used a sexpositive perspective. Most of the studies focused primarily on white populations, and, when people of colour were included, the discourse was usually sex negative. Interestingly, 38\% of the analysed articles focused on sexual orientation, identity and minorities (Hargons et al., 2017). In the author's opinion, the journals of the International Society of Sexual Medicine (Journal of Sexual Medicine, Sexual Medicine Reviews, and Sexual Medicine Open Access) only partially ameliorated this figure and are still primarily concentrated on symptoms and diseases rather than on sexual health from a sex-positive perspective.

Sexuality researchers now have the opportunity to incite change and implement sex-positive values in their interests and practice. There are several benefits in using new approaches to deepen the understanding of human sexuality (Williams et al., 2015). Positive sexuality acknowledges not only the BPS's complex nature of each sexual act, but also the beauty, the uniqueness and the contribution of sexuality in defining the personal identity (Burnes et al., 2017). Researchers working on sexuality have immense power to influence perceptions about what is "normal," healthy, good and remarkable in sexuality and, in the long run, to revise our sexual scripts and meanings (Anderson, 2013). Some pivotal studies reported promising results on the possibility of discussing sexual pleasure and satisfaction to address new programmes, policies and interventions (Gruskin et al., 2019). Holmes et al. (2021) examined the positive outcomes of sexting and the role it plays in intimate relationships in a group of young adults under a sex-positive framework. Some other valuable examples are studies conducted on the efficacy of campaigns such as "PrEP4Love" (Keene et al., 2020) and "\#metoo" (Ison, 2019).

Implementing a sex-positive view does not mean that searching for problems and issues should come to an end. The great advances made in the last few years by researchers should be recognized for increasing our understanding of diverse sexual problems and proposing new treatment options. In this case, being sex-positive means being more effective in addressing negative sexual health outcomes, in recognizing the primary role of sexual distress on symptoms and in capitalizing on the possibilities for improvement (Anderson, 2013).

An urgent sex-positive tool we need to implement in research is the use of a more positive, inclusive and affirmative language when discussing sexual topics. In fact, the use of negative and non-inclusive words instilled with preconceptions, stereotypes and stigma may significantly reinforce internalized sexual negativity (Glickman, 2000). There are many examples of these sex-biases in research, which have led to the stereotyping of phenomena, wrong associations and misinterpretations of data. For example, gender and sexual orientation scripts have guided research on sexual desire and fantasies for years (Nimbi et al., 2020a, 2020b), and literature on HIV/AIDS still focuses primarily on gay men and anal sex (Burnes et al., 2017). A sex-positive approach should change the style of communication by using more updated, inclusive terms and producing more realistic scientific evidence. 
A concrete example is the selection of more appropriate terms and definitions in socio-demographic questionnaires to include a wide range of possibilities and to allow for appropriate descriptions of the variety of gender identities, sexual orientations and possible relational statuses and forms. Another major issue is the focus on penetrative vaginal sex in most of the questionnaires used in the literature, excluding a broader experience of what "having sex" means to people. Moreover, every survey should save space for the participants to describe themselves with their own words through open-ended answers. This is often difficult for researchers who, especially in quantitative studies, need to work with categories, groups, and labels to describe reality in a simplified manner and to easily manage statistical models. Sex-positive research, however, should count on both a quantitative and qualitative understanding of sex and work to heighten the importance of the latter (Ali et al., 2020).

Regarding the topics to be addressed using a sexpositive approach, the choices are numerous. Theoretically, every sexual expression would benefit from being explored under a new "positive" light, but the literature offers some specific suggestions. Berdychevsky and Carr (2020) highlighted the urgency of broadening the BPS perspective of sex as leisure by focusing on sex toys and practices based on consumer perspectives, analysing pleasure in sex/porn addiction, leisure in people with disabilities, rejection and resilience in the LGBTQ + scene, aging; sex tourism, sexual harassment and the complexity of consent. Hibbs (2019) suggested focusing on how policies reflect different cultures and how to create safer spaces in which to report sexual assaults. Sex-positive research needs to integrate social justice and multiculturalism due to the high degree of intersectionality of racism, sexism, heterosexism, cisgenderism, ableism and privilege. The focus should be on the effects of institutionalized and systemic oppression on the experiences of sexuality rather than within- and between-group differences in sexual identity (Mosher, 2017). Kaplan (2014) stressed the need to apply a sex-positive approach in jurisprudence, stating that the law's unspoken assumption is that sexual pleasure has negligible or negative values and that accepting a positive value of sexual pleasure will require a reconceptualization of several regulations. Ali et al. (2020) recommended including more multilevel studies in future research that are able to capture nuances among different ethnic groups and minorities. Moreover, the authors also stress the need to develop a positive understanding of sexuality congruent with cultural, spiritual and religious beliefs. In conclusion, there is a lot to do from a research perspective, starting from reinterpreting the way we think about science, methodologies and sex research.

\section{Clinical Practice}

Most health care providers have recognized the need to move from pathology-based models of sexuality to BPS approaches on well-being. A sex-positive approach is totally in accordance with the ethical principles of many professional associations (ACA, 2005; AMA, 1996; APA, 2010; NASW, 2008) as it recognizes and promotes the needs and choices of each individual (Williams et al., 2015), respecting basic values such as consent, free determination and access to sexual health. Some valuable examples of a clinical integration between the BPS model and the sex-positive approach were presented by Coleman et al. (2018) regarding Impulsive/Compulsive Sexual Behaviour and by Baggett et al. (2017) regarding trauma among sexual assault survivors. However, more work is needed to increase clinicians' comfort and motivation in addressing issues related to sexuality and demonstrating a more sex-positive perspective (Burnes et al., 2017). Sex-positive counselling has the strength to recognize and work on the effects of social inequities, stigma, minority distress and individual attitudes and beliefs (Huang et al., 2010; Mosher, 2017; Sarno et al., 2015). In this sense, Burnes et al. (2017) stated that the sexpositive perspective may offer a powerful tool to clinicians in creating a continuum of attitudes about sexuality from complete sex negativity to sex positivity. Such a continuum could help health care providers understand that individuals rarely identify themselves as completely sex positive or negative: sometimes, they might internalize shame and fear about sex and at other times recognize the enriching power of pleasure. The idea of a continuum of attitudes may facilitate a paradigm shift from a sexual health that mainly focuses on behaviour and towards a more positive understanding that considers sexual identities, emotions, processes and outcomes (Ponzetti, 2015).

A shift of this nature entails an exploration of the many ways that people have internalized sex-negative attitudes and beliefs. This is useful not only for patients, but for clinicians: identifying sex-negative influences among health care providers is the first step to improving their work (Donaghue, 2015). Neglecting personal sexual attitudes can lead clinicians to disconnect with patients, misunderstand sex problems and impose their attitudes on patients. Consequently, sexuality can be overlooked, minimized and even pathologized (Burnes et al., 2017). One of the solutions suggested by authors is to stress the importance of regularly questioning personal beliefs and attitudes, not only in the training phase, but continuously during the clinical career, taking advantage of supervisions and professional updating, which includes an emotional experiential work. In this sense, a sex-positive clinician would be more able to develop sex-positive knowledge and comfort about sexuality, to integrate multiculturalism and social 
justice and to proactively raise sexual topics in care (Cruz et al., 2017; Wandrei, 2019).

Regarding tools for the clinical practice, it is crucial to use a respectful language, recognizing and avoiding discriminatory or devaluing terms and expressions (Williams et al., 2015). One of the best practices is to use patients' words to describe their personal expressions and to rephrase them with more appropriate positive and valuable terms when necessary. Terms such as polyamory, kink and BDSM should enter the health care providers' vocabularies, considering how these constructs may be relevant in clinical practice (Burnes et al., 2017). Regarding relationships, every type of partnership and sexual relationship should be considered valuable, as long as it is based on honest communication, safety and consent among the parties involved (Richards \& Barker, 2013). There is the need to understand patients' specific values and expressions in peculiar forms of relationship, such as open couples and polyamory. This may challenge the idea of a healthy relationship that considers only monogamous couples based on traditional psychological approaches, which, if they are too rigid, may negatively influence the client-therapist relationship (Burnes et al., 2017). For example, in these cases, the clinician should facilitate the discussion of the partners' values and needs regarding relationships and sexuality, emphasizing the recognition of diversities and supporting the partners to find their own balance, which can be constituted by unique compromises and explicit rules; if it is not possible to find another type of agreement that is satisfactory for both parties, the clinician should then fairly discuss the possibility of terminating the relationship.

Partners may engage in less commonly documented forms of sexual expressions and behaviours that health care professionals need to be aware of and understand. Regarding BDSM and kinky practices, Burnes et al. (2017) underlined the need for clinicians to recognize this range of experiences and familiarize themselves with the related communities. Common sex-negative assumptions about BDSM include beliefs that these behaviours are a result of traumatic experiences (especially in childhood) and that those who take on submissive roles are powerless and distressed. Scientific evidence has highlighted how BDSM practitioners inserted in a community where their fantasies and behaviours are accepted and shared reported higher sexual satisfaction and well-being than the general population (Botta et al., 2019). However, many people practicing kinky behaviours feel significant shame and distress related to the widespread stigma and vilification of the practices (Burnes et al., 2017; Yost \& Hunter, 2012). Thus, a sexpositive clinician should be able to reduce this distress by working out the potential strengths of these practices (Joyal et al., 2015).

\section{Professional Training}

Sexuality courses offered within professional training programmes often focus on sexual dysfunctions, pathology, infectious diseases and related treatments (Mosher, 2017). One of the positive effects of these courses is that professionals who have attended continuing education programmes on human sexuality are usually more likely to ask patients about sexuality than other clinicians, although they focus primarily on pathology (Miller \& Byers, 2010). To reach a higher standard in sexological training, authors have emphasized the importance of working on personal attitudes and developing a self-awareness of sex and sexuality (Mosher, 2017; Nimbi et al., 2020c). Training sessions focus on restructuring beliefs, awareness, sex attitudes and skills needed to incorporate multicultural constructs such as intersectionality and disability, as well as historical aspects of the construction of gender and sexuality (Huang et al., 2010; Mosher, 2017; Sarno et al., 2015). For example, an Italian study on clinicians who attended educational programmes in sexology that devoted part of the course on working on sexual attitudes and personal emotions related to sex reported that these individuals demonstrated more positive attitudes towards lesbian and gay marriage and parenthood than other health care professionals (Nimbi et al., 2020c).

Antebi-Gruszka et al. (2019) suggested some sex-positive guidelines for clinicians taking care of sex workers that, in our opinion, could be easily extended to the general population. The authors stressed the importance of addressing and enhancing health care professionals' self-awareness of their own biases about sexuality and behaviour, providing useful information about the multifaceted experiences of their patients and how to put culturally relevant counselling skills and intervention strategies into practice to improve patients' sexual health and QoL.

Prior et al. (2016) highlighted the limited presence of sexpositive content in social work curricula. They suggested how discussing sexuality directly within "Human Behaviour and the Social Environment" classes may provide useful skills for students when implementing positive sexual messages in their own practice. Dodd and Katz (2020) suggested some concrete strategies for creating a sex-positive environment within professional courses such as regularly discussing (and not only in designed moments) sexuality and all of its possible expressions, as well as the various sexual orientations and identities, and including the discussion of sexual aspects in clinical cases. Breaking the silence around sexuality in professional courses gives future health care providers permission to do the same in their practice (Sitron \& Dyson, 2012). The central role of the sexual dimension should be emphasized when the BPS is applied, providing relevant examples of inclusive and non-assumptive questions (e.g. not assuming monogamy, gender, sexual orientation or 
number of partners). Sexuality should be addressed appropriately in accordance with one's age; clinicians must recognize the positive role it may play in different stages of life and use relevant content and language for certain age groups (e.g. children, adolescents, elders). Moreover, the training of every health care professional should encourage the use of correct terms and address important topics related to selfdetermination, such as consent, pleasure, desire, choice, decision making and agency.

\section{Sex Education}

The sex-positive approach provides an opportunity to deal with sex education under a new light and in more inclusive, open and multidisciplinary ways (Williams et al., 2013, 2015). Many international guidelines (IPPF, 2016; IPPF \& WAS, 2016; SIECCAN, 2019; UNESCO, 2019; WHO, 2010,2018 ) agree on the need for a sex-positive comprehensive education, which is recognized as the best practice for children and young people to empower themselves, take control and make informed decisions about their sexuality and relationships freely and responsibly. But speaking about positive aspects of sexuality with young people is never easy.

The right to pleasure has the potential to achieve safer sexual health outcomes and contribute to gender equality (Hirst, 2013). However, it is difficult to discuss pleasure in current practice since the topic can be scary at different levels. A discussion of the history of fearing pleasure goes far beyond the objectives of the current review. In this sense, we refer to previous works that deal with this phenomenon in detail (Berdychevsky \& Carr, 2020; Hirst, 2013; Wood et al., 2019). Here, we are concerned with the enriching aspects that a comprehensive sex education with a positive and inclusive orientation towards pleasure can bring. A successful and healthy sexual experience should involve feeling in control regarding the sexual act, without feeling regret or worrying about having contracted an STI and/or conception (Hirst, 2013). In this regard, the emphasis on the negative consequences of sex for health is not enough to promote safer sex. Nimbi et al. (2019) highlighted that contraception is more effective when it involves clear and open communication on sex. Moreover, there is the opportunity for sex education that endorses pleasure to challenge gendered socio-cultural expectations of heteronormativity that favours the silence around female pleasure (Berdychevsky $\&$ Carr, 2020). According to this perspective, an effective sex-positive education should create a safe space for discussing consent, gender identity and roles, criticizing the scripts of mainstream pornography and talking about equality in relationships, among other topics. Hirst (2013) stated that when pleasure is asserted as a right and reinforced through communication and sex education, young people seem to be more likely to refer to their own feelings and decline pressures of unwanted sex.

However, caution is recommended against creating and reinforcing a "pleasure imperative" (Wood et al., 2019) that may set a benchmark to compare the success/unsuccess of any sexual experience and reinforce a performative view of sexual experience. Not every sexual experience is pleasurable, and it should be stressed that less positive experiences are also fine (apart from unwanted and coerced acts). The real challenge for sex educators is to find a balance between providing an informed and comprehensive positive approach to sexuality and recognize the cis-gendered, heteronormative relations and cultures that usually contextualize our daily lives. In this sense, it is essential for sex educators to have structured training that allows them to work on their attitudes, motivation and ability to communicate sexual content to young people and stakeholders (Hirst, 2013).

A sex-positive discussion of sex education is applicable across different contexts and actors. For example, parents have a unique opportunity to instil knowledge as well as confidence around sexuality. Although parent-child communication about sexual risk is common, less is known regarding the frequency of parent-child communication about sex-positive topics. Evans et al. (2020) confronted the frequency of parent-children communication about sexual risk and positive topics across the USA. Results showed that few parents communicated with their adolescents about sexpositive topics: $38 \%$ about sexual satisfaction, $38 \%$ about different types of practices (e.g. oral sex) and 55\% about desire. As expected, parents, especially mothers and fathers of daughters, reported communicating more about risks in sexual behaviours than sex-positive topics. However, many parents expressed their will to learn how to talk with their children about sex in a way that makes them feel happy, healthy and good in their bodies and help them set good boundaries (Gubrium \& Shafer, 2014).

Another important element concerns sex education and health communication in informal contexts. Sex-positive approaches in sex education through media communication for adolescents and young adults have become increasingly popular (Harden, 2014). Some studies have highlighted the positive and negative reactions of this kind of action (Brickman \& Willoughby, 2017; Hirshfield et al., 2019; Hovick \& Silver, 2019; Keene et al., 2020; Raymond \& Hutchinson, 2019). Hirshfield et al. (2019) reported the risk reduction results of a video-based web intervention on Men who have Sex with Men (MSM) within a sex-positive framework, while Hovick and Silver (2019) showed how students paid attention and reacted positively to consent poster campaigns on a university campus using a sex-positive approach. Brickman and Willoughby (2017) focused on the reactions of young adults to positive or negative sex-related text messages used in a telephone-based intervention. Positive messages were contextualized as gain 
frames and were preferred over negative/loss texts and experienced as more believable and persuasive. The authors suggested that sex-positive messages that focus on the benefits of specific behaviours tend to resonate better with young adults. However, it should be considered that sex-positive campaigns are not always welcomed in every context. Keene et al. (2020) discussed the critiques and the negative responses to the aforementioned "PrEP4Love" campaign, a sex-positive inclusive project launched online and in public spaces. Advertisements prominently featured black sexual minority men and transgender women who were strategically placed in diverse neighbourhoods to generate a wider and differentiated reaction from the population. The study discussed the negative responses to the project, including viewers' belief that the project was too sexually explicit, their fear of its adverse effects on children, how it negatively depicted black homosexuality, general antiLGBTQ + comments and the general stigmatization of racial minorities. Despite some negative responses, the "PrEP4Love" campaign increased awareness and improved the engagement of Pre-Exposure Prophylaxis (PrEP) overall. However, the discussion of negative reactions to this kind of campaign is fundamental to develop future sexual health promotion strategies that are able to engage with, and be effective in, such complex and multivariate contexts, especially considering that the sexpositive approach was born and continues to gain relevance as a social and activist movement.

\section{Conclusions}

After 45 years from the first general formulation of the BPS model (Engels, 1977), its implementation in the sexual health field can still be sensibly improved under different aspects, particularly in regard to the socio-cultural factors involved. The possibility of incorporating a sex-positive approach into the BPS model may represent an interesting new challenge for every health care professional. The sexpositive approach, in our opinion, represents a viable path that entails the willingness of health care professionals to get involved actively; criticize their personal attitudes, beliefs and knowledge about sex and work hard to improve their practice in sexology.

This may be the time to be brave in science. Available literature has widely shown that sexual satisfaction, pleasure and positive sexual self-esteem may significantly improve sexual health as well as mental and physical health outcomes (Anderson, 2013). The growing attention on how sexuality may positively impact QoL is an important shift in the discourse of sexual health. The sex-positive approach emphasizes that sexuality is a vital aspect of the clinical process and is a central part of a healthy development, QoL and resilience over the course of one's life. The core values of social justice, resilience and wellness provide a rationale for health care professional to become protagonists in including sex-positive frameworks into their research, practice, training and supervision in order to work more holistically with their patients (Burnes et al., 2017). Some strategies were discussed in this review, but more studies on the implementation of a sex-positive approach in already existing BPS realities are strongly needed to spread better and more precise clinical practices.

We would like to underline how openness to a more positive sexuality in the fields of research, clinical practice, professional training and sex education may foster a real cultural and health revolution that produces new knowledge, new practices and new possibilities; increases access and engagement to services and enriches professional skills to improve QoL and patient health. The road ahead is still long and winding, but perhaps the time has never been better to invest in health promotion.

Author Contribution NFM, JEA, SC and TR originated the idea and design for the current study. LE, GC and FL conducted literature searches and provided summaries of previous research studies. NMF, GR and RR wrote the first draft of the manuscript. NMF, JEA, $\mathrm{SC}$ and TR supervised the research process and the final draft of the manuscript. All the authors contributed to and have approved the final manuscript.

Funding Open access funding provided by Università degli Studi di Roma La Sapienza within the CRUI-CARE Agreement.

Availability of Data and Material Not applicable.

Code Availability Not applicable.

\section{Declarations}

Competing Interests The authors declare no competing interests.

Open Access This article is licensed under a Creative Commons Attribution 4.0 International License, which permits use, sharing, adaptation, distribution and reproduction in any medium or format, as long as you give appropriate credit to the original author(s) and the source, provide a link to the Creative Commons licence, and indicate if changes were made. The images or other third party material in this article are included in the article's Creative Commons licence, unless indicated otherwise in a credit line to the material. If material is not included in the article's Creative Commons licence and your intended use is not permitted by statutory regulation or exceeds the permitted use, you will need to obtain permission directly from the copyright holder. To view a copy of this licence, visit http://creativecommons.org/licenses/by/4.0/.

\section{References}

American Counseling Association. (2005). ACA code of ethics. Alexandria, VA: Author. 
Ali, S., Keo, B. S., \& Chaudhuri, S. (2020). Critically understanding South Asian sexual health: A call for a holistic and sex positive approach. International Journal of Sexual Health, 1-11.

Althof, S. E. (2007). It was the best of times; it was the worst of times. Journal of Sex \& Marital Therapy, 33(5), 399-403.

Althof, S. E., \& Needle, R. B. (2011). Psychological factors associated with male sexual dysfunction: Screening and treatment for the urologist. Urologic Clinics of North America, 38(2), 141-146.

AMA - American Medical Association. . (1996). AMA code of medical ethics. Author.

Anderson, R. M. (2013). Positive sexuality and its impact on overall well-being. Bundesgesundheitsblatt-GesundheitsforschungGesundheitsschutz, 56(2), 208-214.

Antebi-Gruszka, N., Spence, D., \& Jendrzejewski, S. (2019). Guidelines for mental health practice with clients who engage in sex work. Sexual and Relationship Therapy, 34(3), 339-354.

American Psychological Association. (2010). Ethical principles of psychologists and code of conduct. Author.

Arakawa, D. R., Flanders, C. E., Hatfield, E., \& Heck, R. (2013). Positive psychology: What impact has it had on sex research publication trends? Sexuality \& Culture, 17(2), 305-320.

Atallah, S., Johnson-Agbakwu, C., Rosenbaum, T., Abdo, C., Byers, E. S., Graham, C., \& Brotto, L. (2016). Ethical and sociocultural aspects of sexual function and dysfunction in both sexes. The Journal of Sexual Medicine, 13(4), 591-606.

Aumer, K. (2014). The influence of culture and gender on sexual self-schemas and satisfaction in romantic relationships. Sexual and Relationship Therapy, 29(3), 280-292.

Baggett, L. R., Eisen, E., Gonzalez-Rivas, S., Olson, L. A., Cameron, R. P., \& Mona, L. R. (2017). Sex-positive assessment and treatment among female trauma survivors. Journal of Clinical Psychology, 73(8), 965-974.

Berdychevsky, L., \& Carr, N. (2020). Innovation and impact of sex as leisure in research and practice: Introduction to the special issue. Leisure Sciences, 1-20.

Berry, M. D., \& Berry, P. D. (2013). Contemporary treatment of sexual dysfunction: Reexamining the biopsychosocial model. The Journal of Sexual Medicine, 10(11), 2627-2643.

Bhugra, D., Popelyuk, D., \& McMullen, I. (2010). Paraphilias across cultures: Contexts and controversies. Journal of Sex Research, 47(2-3), 242-256.

Botta, D., Nimbi, F. M., Tripodi, F., Silvaggi, M., \& Simonelli, C. (2019). Are role and gender related to sexual function and satisfaction in men and women practicing BDSM? The Journal of Sexual Medicine, 16(3), 463-473.

Boyer, S., Chamberlain, S., \& Pukall, C. (2017). Vulvodynia attitudes in a sample of Canadian post-graduate medical trainees. The Canadian Journal of Human Sexuality, 26(3), 249-260.

Brickman, J., \& Willoughby, J. F. (2017). 'You shouldn't be making people feel bad about having sex': Exploring young adults' perceptions of a sex-positive sexual health text message intervention. Sex Education, 17(6), 621-634.

Bullough, V. L. (1976). Sexual variance in society and history. John Wiley \& Sons.

Burnes, T. R., Singh, A. A., \& Witherspoon, R. G. (2017). Sex positivity and counseling psychology: An introduction to the major contribution. The Counseling Psychologist, 45(4), 470-486.

Casagrande, S. S., Gary, T. L., LaVeist, T. A., Gaskin, D. J., \& Cooper, L. A. (2007). Perceived discrimination and adherence to medical care in a racially integrated community. Journal of General Internal Medicine, 22(3), 389-395.

Coleman, E., Dickenson, J. A., Girard, A., Rider, G. N., CandelarioPérez, L. E., Becker-Warner, R., \& Munns, R. (2018). An integrative biopsychosocial and sex positive model of understanding and treatment of impulsive/compulsive sexual behavior. Sexual Addiction \& Compulsivity, 25(2-3), 125-152.
Coleman, E., Elders, J., Satcher, D., Shindel, A., Parish, S., Kenagy, G., \& Light, A. (2013). Summit on medical school education in sexual health: Report of an expert consultation. The Journal of Sexual Medicine, 10(4), 924-938.

Coyne, S. M., Ward, L. M., Kroff, S. L., Davis, E. J., Holmgren, H. G., Jensen, A. C., \& Essig, L. W. (2019). Contributions of mainstream sexual media exposure to sexual attitudes, perceived peer norms, and sexual behavior: A meta-analysis. Journal of Adolescent Health, 64(4), 430-436.

Cruz, C., Greenwald, E., \& Sandil, R. (2017). Let's talk about sex: Integrating sex positivity in counseling psychology practice. The Counseling Psychologist, 45(4), 547-569.

Dodd, S. J., \& Katz, C. C. (2020). Sex positive social work education: Integrating content into HBSE courses and beyond. Journal of Teaching in Social Work, 40(1), 48-57.

Donaghue, C. (2015). Sex outside the lines: Authentic sexuality in a sexually dysfunctional culture. Benbella Books.

Durso, L. E., \& Meyer, I. H. (2013). Patterns and predictors of disclosure of sexual orientation to healthcare providers among lesbians, gay men, and bisexuals. Sexuality Research and Social Policy, 10(1), 35-42.

Earnshaw, V. A., Jin, H., Wickersham, J., Kamarulzaman, A., John, J., \& Altice, F. L. (2014). Exploring intentions to discriminate against patients living with HIV/AIDS among future healthcare providers in Malaysia. Tropical Medicine \& International Health, 19(6), 672-679.

Eichenberg, C., Khamis, M., \& Hübner, L. (2019). The attitudes of therapists and physicians on the use of sex robots in sexual therapy: online survey and interview study. Journal of medical Internet research, 21(8), e13853.

Engel, G. L. (1977). The need for a new medical model: A challenge for biomedicine. Science, 196, 129-136.

Evans, R., Widman, L., Kamke, K., \& Stewart, J. L. (2020). Gender differences in parents' communication with their adolescent children about sexual risk and sex-positive topics. The Journal of Sex Research, 57(2), 177-188.

Fahs, B. (2014). 'Freedom to' and 'freedom from': A new vision for sex-positive politics. Sexualities, 17(3), 267-290.

Fisher, A. D., Castellini, G., Ristori, J., Casale, H., Giovanardi, G., Carone, N., \& Maggi, M. (2017). Who has the worst attitudes toward sexual minorities? Comparison of transphobia and homophobia levels in gender dysphoric individuals, the general population and health care providers. Journal of Endocrinological Investigation, 40(3), 263-273.

Frederick, N. N., Campbell, K., Kenney, L. B., Moss, K., Speckhart, A., \& Bober, S. L. (2018). Barriers and facilitators to sexual and reproductive health communication between pediatric oncology clinicians and adolescent and young adult patients: The clinician perspective. Pediatric blood \& cancer, 65(8), e27087.

Gewirtz-Meydan, A., Even-Zohar, A., \& Werner, S. (2018). Examining the attitudes and knowledge of social work and nursing students on later-life sexuality. Canadian Journal on Aging/la Revue Canadienne Du Vieillissement, 37(4), 377-389.

Glickman, C. (2000). The language of sex positivity. Electronic Journal of Human Sexuality, 3, 1-5.

Goldstein, I. (2012). Sexual medicine reflects the light of knowledge. The Journal of Sexual Medicine, 9(11), 2733-2735.

Grunt-Mejer, K. (2021). The history of the medicalisation of rapid ejaculation-A reflection of the rising importance of female pleasure in a phallocentric world. Psychology \& Sexuality, 1-18.

Gruskin, S., Yadav, V., Castellanos-Usigli, A., Khizanishvili, G., \& Kismödi, E. (2019). Sexual health, sexual rights and sexual pleasure: Meaningfully engaging the perfect triangle. Sexual and Reproductive Health Matters, 27(1), 29-40. 
Gubrium, A. C., \& Shafer, M. B. (2014). Sensual sexuality education with young parenting women. Health Education Research, 29(4), 649-661.

Harden, K. P. (2014). A sex-positive framework for research on adolescent sexuality. Perspectives on Psychological Science, 9(5), 455-469.

Hargons, C., Mosley, D. V., \& Stevens-Watkins, D. (2017). Studying sex: A content analysis of sexuality research in counseling psychology. The Counseling Psychologist, 45(4), 528-546.

Hatzichristou, D., Kirana, P. S., Banner, L., Althof, S. E., Lonnee-Hoffmann, R. A., Dennerstein, L., \& Rosen, R. C. (2016). Diagnosing sexual dysfunction in men and women: Sexual history taking and the role of symptom scales and questionnaires. The Journal of Sexual Medicine, 13(8), 1166-1182.

Herek, G. M., Gillis, J. R., \& Cogan, J. C. (2009). Internalized stigma among sexual minority adults: Insights from a social psychological perspective. Journal of Counseling Psychology, 56(1), 32.

Hibbs, L. E. (2019). Regulation and reporting: A study of policy-based inhibition of sex-positive cultures and sexual assault reporting on college campuses.

Hirshfield, S., Downing, M. J., Chiasson, M. A., Yoon, I. S., Houang, S. T., Teran, R. A., \& Parsons, J. T. (2019). Evaluation of sex positive! A video eHealth intervention for men living with HIV. AIDS and Behavior, 23(11), 3103-3118.

Hirst, J. (2013). 'It's got to be about enjoying yourself': Young people, sexual pleasure, and sex and relationships education. Sex Education, 13(4), 423-436.

Hoff, G., \& Sprott, R. A. (2009). Therapy experiences of clients with BDSM sexualities: Listening to a stigmatized sexuality. Electronic Journal of Human Sexuality, 12(9), 30.

Holmes, L. G., Nilssen, A. R., Cann, D., \& Strassberg, D. S. (2021) A sex-positive mixed methods approach to sexting experiences among college students. Computers in Human Behavior, 115, 106619.

Hovick, S. R., \& Silver, N. (2019). "Consent is sexy": A poster campaign using sex-positive images and messages to increase dyadic sexual communication. Journal of American College Health, 67(8), 817-824.

Huang, Y., Brewster, M. E., Moradi, B., Goodman, M. B., Wiseman, M. C., \& Martin, A. (2010). Content analysis of literature about LGB people of color: 1998-2007. The Counseling Psychologist, 38, 363-396.

International Planned Parenthood Federation. (2016). Putting sexuality back into comprehensive sexuality education: Making the case for a rights-based, sex-positive approach. Last accessed $18^{\text {th }}$ March 2021 at: https://www.ippf.org/resource/putting-sexuality-backcomprehensive-sexuality-education

International Planned Parenthood Federation \& WAS - World Association for Sexual Health. (2016). Fulfil! Guidance document for the implementation of young people's sexual rights. Last accessed 26th July 2021 at: https://www.ippf.org/resource/fulfil-guidancedocument-implementation-young-peoples-sexual-rights-ippf-was

Ison, J. (2019). 'It's not just men and women': LGBTQIA people and\# MeToo. In \# MeToo and the politics of social change (pp. 151167). Palgrave Macmillan, Cham.

Ivanski, C., \& Kohut, T. (2017). Exploring definitions of sex positivity through thematic analysis. The Canadian Journal of Human Sexuality, 26(3), 216-225.

Jannini, E. A. (2017). SM= SM: The interface of systems medicine and sexual medicine for facing non-communicable diseases in a genderdependent manner. Sexual Medicine Reviews, 5(3), 349-364.

Jannini, E. A., \& Reisman, Y. (2019). Medicine without sexual medicine is not medicine: An MJCSM and ESSM petition on sexual health to the political and university Authorities. The Journal of Sexual Medicine, 16(6), 943-945.
Jannini, E. A., Limoncin, E., Ciocca, G., Buehler, S., \& Krychman, M. (2012). Ethical aspects of sexual medicine. Internet, vibrators, and other sex aids: Toys or therapeutic instruments. The journal of sexual medicine, 9(12), 2994-3001.

Jannini, E. A., McCabe, M. P., Salonia, A., Montorsi, F., \& Sachs, B. D. (2010). Controversies in sexual medicine: Organic vs. psychogenic? The Manichean diagnosis in sexual medicine. The journal of sexual medicine, 7(5), 1726-1733.

Joyal, C. C., Cossette, A., \& Lapierre, V. (2015). What exactly is an unusual sexual fantasy? The Journal of Sexual Medicine, 12(2), $328-340$.

Kaplan, M. (2014). Sex-Positive Law. NYUL Rev., 89, 89.

Katz, A. (2005). Do ask, do tell: Why do so many nurses avoid the topic of sexuality? AJN the American Journal of Nursing, 105(7), 66-68.

Keene, L. C., Dehlin, J. M., Pickett, J., Berringer, K. R., Little, I., Tsang, A., \& Schneider, J. A. (2020). \# PrEP4Love: Success and stigma following release of the first sex-positive PrEP public health campaign. Culture, Health \& Sexuality, 1-17.

Kimmes, J. G., Mallory, A. B., Cameron, C., \& Köse, Ö. (2015). A treatment model for anxiety-related sexual dysfunctions using mindfulness meditation within a sex-positive framework. Sexual and Relationship Therapy, 30(2), 286-296.

Kirana, S. P., Tripodi, F., Reisman, Y., \& Porst, H. (2013). The EFS and ESSM Syllabus of Clinical Sexology. Medix.

Klaeson, K., Hovlin, L., Guvå, H., \& Kjellsdotter, A. (2017). Sexual health in primary health care-a qualitative study of nurses' experiences. Journal of Clinical Nursing, 26(11-12), $1545-1554$.

Klein, V., Imhoff, R., Reininger, K. M., \& Briken, P. (2019). Perceptions of sexual script deviation in women and men. Archives of Sexual Behavior, 48(2), 631-644.

Kleinplatz, P. J. (2013). Three decades of sex: Reflections on sexuality and sexology. Canadian Journal of Human Sexuality, 2013;22(1):1-4.

Kolmes, K., Stock, W., \& Moser, C. (2006). Investigating bias in psychotherapy with BDSM clients. Journal of Homosexuality, 50(2/3), 301-324.

Kotronoulas, G., Papadopoulou, C., \& Patiraki, E. (2009). Nurses' knowledge, attitudes, and practices regarding provision of sexual health care in patients with cancer: Critical review of the evidence. Supportive Care in Cancer, 17(5), 479-501.

Levin, R. J. (2020). The clitoris-An appraisal of its reproductive function during the fertile years: Why was it, and still is, overlooked in accounts of female sexual arousal. Clinical Anatomy, 33(1), 136-145.

Martino, A. S., \& Perreault-Laird, J. (2019). " I don't know if I can talk about that": An exploratory study on the experiences of care workers regarding the sexuality of people with intellectual disabilities. Disability Studies Quarterly, 39(3).

Maxwell, J. A., Muise, A., MacDonald, G., Day, L. C., Rosen, N. O., \& Impett, E. A. (2017). How implicit theories of sexuality shape sexual and relationship well-being. Journal of Personality and Social Psychology, 112(2), 238.

Miller, S. A., \& Byers, E. S. (2010). Psychologists' sexual education and training in graduate school. Canadian Journal of Behavioural Science, 42, 93-100.

Miranda, E. P., Taniguchi, H., Cao, D. L., Hald, G. M., Jannini, E. A., \& Mulhall, J. P. (2019). Application of sex aids in men with sexual dysfunction: A review. The Journal of Sexual Medicine, 16(6), 767-780.

Monteiro, A., von Humboldt, S., \& Leal, I. (2017). How do formal caregivers experience the sexuality of older adults? Beliefs and attitudes towards older adults' sexuality.

Montorsi, F., \& Althof, S. E. (2004). Partner responses to sildenafil citrate (Viagra) treatment of erectile dysfunction. Urology, 63(4), $762-767$. 
Moscatelli, A., Nimbi, F. M., Ciotti, S., \& Jannini, E. A. (2020). Haptic and somesthetic communication in sexual medicine. Sexual Medicine Reviews.

Mosher, C. M. (2017). Historical perspectives of sex positivity: Contributing to a new paradigm within counseling psychology. The Counseling Psychologist, 45(4), 487-503.

NASW - National Association of Social Workers. (2008). Code of ethics. Author.

Neculăesei, A. N. (2015). Culture and gender role differences. CrossCultural Management Journal, 17(01), 31-35.

Nimbi, F. M., Ciocca, G., Limoncin, E., Fontanesi, L., Uysal, Ü. B., Flinchum, M., \& Simonelli, C. (2020a). Sexual desire and fantasies in the LGBT+ community: Focus on lesbian women and gay men. Current Sexual Health Reports, 1-9.

Nimbi, F. M., Ciocca, G., Limoncin, E., Fontanesi, L., Uysal, Ü. B., Flinchum, M., \& Simonelli, C. (2020b). Sexual desire and fantasies in the LGBT+ community: A focus on bisexuals, transgender, and other shades of the rainbow. Current Sexual Health Reports, 1-8.

Nimbi, F. M., Rossi, R., Tripodi, F., Wylie, K., \& Simonelli, C. (2019). A biopsychosocial model for the counseling of hormonal contraceptives: A review of the psychological, relational, sexual, and cultural elements involved in the choice of contraceptive method. Sexual Medicine Reviews, 7(4), 587-596.

Nimbi, F. M., Tripodi, F., Rossi, R., \& Simonelli, C. (2018). Testing a conceptual model for men's sexual desire referring to automatic thoughts, emotions, sexual function, and sexism. The Journal of Sexual Medicine, 15(11), 1518-1526.

Nimbi, F. M., Viozzi, E., Tripodi, F., Simonelli, C., \& Baiocco, R. (2020c). Could educational programs in sexology have an influence on attitudes towards same-sex marriage and parenting? Sexologies, 29(3), e77-e83.

Parish, S. J., \& Rubio-Aurioles, E. (2010). Education in sexual medicine: Proceedings from the international consultation in sexual medicine, 2009. The Journal of Sexual Medicine, 7(10), 3305-3314.

Perelman, M. A. (2015). Advocating for a transdisciplinary perspective in sexual medicine. Current Sexual Health Reports, 7(1), 1-2.

Petroll, A. E., \& Mosack, K. E. (2011). Physician awareness of sexual orientation and preventive health recommendations to men who have sex with men. Sexually Transmitted Diseases, 38(1), 63.

Ponzetti Jr, J. J. (2015). Future trends and directions in sexuality education. In Evidence-based Approaches to Sexuality Education (pp. 383-392). Routledge.

Prior, E., Williams, D. J., Zavala, T., \& Milford, J. (2016). What do (n't) American undergraduate social work students learn about sex? A content analysis of sex positivity and diversity in five popular HBSE Textbooks. Critical Social Work, 17(1).

Raymond, N. M., \& Hutchison, A. N. (2019). A pilot test of the effectiveness of an integrated sex positive education program. American Journal of Sexuality Education, 14(3), 315-341.

Reisman, Y., Porst, H., Lowenstein, L., Tripodi, F., \& Kirana, S. P. (2015). The ESSM Manual of Sexual Medicine. Medix.

Richards, C., \& Barker, M. (2013). Sexuality and gender for mental health professionals: A practical guide. Sage.

Sabin, J. A., Riskind, R. G., \& Nosek, B. A. (2015). Health care providers' implicit and explicit attitudes toward lesbian women and gay men. American Journal of Public Health, 105(9), 1831-1841.

Sarno, E. L., Mohr, J. J., Jackson, S. D., \& Fassinger, R. E. (2015). When identities collide: Conflicts in allegiances among LGB people of color. Cultural Diversity and Ethnic Minority Psychology, 21, 550-559.

Sex Information \& Education Council of Canada. (2019). Canadian Guidelines for sexual health. Last accessed $18^{\text {th }}$ March 2021 at: https://www.actioncanadashr.org/resources/factsheets-guidelines/ 2019-06-25-canadian-guidelines-sexual-health-education
Simonelli, C., Fabrizi, A., Rossi, R., Silvaggi, C., Tripodi, F., \& Michetti, P. M. (2010). Clinical sexology: An integrated approach between the psychosomatic and the somatopsychic. Sexologies, 19(1), 3-7.

Sitron, J. A., \& Dyson, D. A. (2012). Validation of sexological worldview: A construct for use in the training of sexologists in sexual diversity. SAGE Open, 2(1), 2158244012439072.

Tamas, D., Jovanovic, N. B., Rajic, M., Ignjatovic, V. B., \& Prkosovacki, B. P. (2019). Professionals, parents and the general public: Attitudes towards the sexuality of persons with intellectual disability. Sexuality and Disability, 37(2), 245-258.

Tavares, I. M., Moura, C. V., \& Nobre, P. J. (2020). The role of cognitive processing factors in sexual function and dysfunction in women and men: a systematic review. Sexual medicine reviews.

Tracy, J. K., Lydecker, A. D., \& Ireland, L. (2010). Barriers to cervical cancer screening among lesbians. Journal of Women's Health, 19(2), 229-237.

Træen, B., Carvalheira, A. A., Hald, G. M., Lange, T., \& Kvalem, I. L. (2019). Attitudes towards sexuality in older men and women across Europe: Similarities, differences, and associations with their sex lives. Sexuality \& Culture, 23(1), 1-25.

Tsimtsiou, Z., Hatzimouratidis, K., Nakopoulou, E., Kyrana, E., Salpigidis, G., \& Hatzichristou, D. (2006). Education: Predictors of physicians' involvement in addressing sexual health issues. The Journal of Sexual Medicine, 3(4), 583-588.

United Nations Educational, Scientific and Cultural Organization. (2019). International technical guidance on sexuality education. An evidence-informed approach. Last accessed $18^{\text {th }}$ March 2021 at: https://www.unfpa.org/sites/default/files/pub-pdf/ITGSE.pdf

Vijay, A., Earnshaw, V. A., Tee, Y. C., Pillai, V., White Hughto, J. M., Clark, K., \& Wickersham, J. A. (2018). Factors associated with medical doctors' intentions to discriminate against transgender patients in Kuala Lumpur. Malaysia. LGBT Health, 5(1), 61-68.

Wandrei, K. E. (2019). An unintended consequence of online directories for people seeking sex-positive psychotherapists. Journal of Positive Sexuality, 5(2).

World Association for Sexual Health. (2014). Declaration of sexual rights. Last accessed $18^{\text {th }}$ March 2021 at: https://worldsexualhealth.net/wpcontent/uploads/2013/08/Declaration-of-Sexual-Rights-2014-plaintext.pdf

World Association for Sexual Health. (2019). Declaration on sexual pleasure. Last accessed $26^{\text {th }}$ July 2021 at: https://worldsexualhealth.net/ wp-content/uploads/2019/10/2019_WAS_Declaration_on_Sexual_ Pleasure.pdf

World Health Organization. (2006). Defining sexual health: Report of a technical consultation on sexual health. Geneva, Switzerland: Author. Last accessed $18^{\text {th }}$ March 2021 at: https://www.who.int/ reproductivehealth/publications/sexual_health/defining_sh/en/

World Health Organization. (2010). WHO Regional Office for Europe and BZgA Standards for Sexuality Education in Europe. A framework for policy makers, educational and health authorities and specialists. Last accessed $18^{\text {th }}$ March 2021 at: https://www. bzga-whocc.de/fileadmin/user_upload/WHO_BZgA_Standards_ English.pdf

World Health Organization. (2018). WHO recommendations on adolescent sexual and reproductive health and rights. Last accessed $18^{\text {th }}$ March 2021 at: https://apps.who.int/iris/bitstream/handle/ 10665/275374/9789241514606-eng.pdf?ua=1

Williams, D. J., Prior, E., \& Wegner, J. (2013). Resolving social problems associated with sexuality: Can a "sex-positive" approach help? Social Work, 58(3), 273-276.

Williams, D. J., Thomas, J. N., Prior, E. E., \& Walters, W. (2015). Introducing a multidisciplinary framework of positive sexuality. Journal of Positive Sexuality, 1(6), 6-11. 
Wood, R., Hirst, J., Wilson, L., \& Burns-O’Connell, G. (2019). The pleasure imperative? Reflecting on sexual pleasure's inclusion in sex education and sexual health. Sex Education, 19(1), 1-14.

Yost, M. R., \& Hunter, L. E. (2012). BDSM practitioners' understandings of their initial attraction to BDSM sexuality: Essentialist and constructionist narratives. Psychology \& Sexuality, 3(3), 244-259.
Publisher's Note Springer Nature remains neutral with regard to jurisdictional claims in published maps and institutional affiliations. 